\title{
Hidratação enteral em bovinos: avaliação de soluções eletrolíticas isotônicas administradas por sonda nasogástrica em fluxo contínuo
}

\author{
Enteral fluid therapy in cattle: evaluation of isotonic electrolyte solutions administered \\ via nasogastric tube on continuous flow
}

\section{José Dantas Ribeiro Filho ${ }^{\mathrm{I}}$ Angélica Misailidis Gimenes $^{\mathrm{I}}$ Eduardo Ferreira Fonseca $^{\mathrm{I}}$ Waleska de Melo Ferreira Dantas ${ }^{\mathrm{I}}$ Tânia Toledo de Oliveira ${ }^{\mathrm{II}}$}

RESUMO

\begin{abstract}
Foram avaliados os efeitos de soluções eletrolíticas isotônicas administradas via sonda nasogástrica de pequeno calibre e fluxo contínuo sobre o hematócrito, proteína total, albumina, sódio, potássio, cloreto, magnésio total, uréia, creatinina, glicose e lactato de bovinos desidratados experimentalmente. Seis bovinos adultos, mestiços, dois machos e quatro fêmeas foram avaliados. A hidratação enteral foi administrada na dose de $15 \mathrm{~mL} \mathrm{~kg}^{-1} \mathrm{~h}^{-1}$ durante 12 horas. Os animais foram separados em dois tratamentos num delineamento experimental crossover e submetidos a cada um dos seguintes tratamentos: SEGlic: $\mathrm{NaCl} 4 \mathrm{~g}, \mathrm{KCl} 1 \mathrm{~g}$, $\mathrm{NaHCO}$ $4 \mathrm{~g}$, dextrose $5 \mathrm{~g}$ em 1.000mL de água; SEProp: $\mathrm{NaCl} 8 \mathrm{~g}, \mathrm{KCl}$ $0,5 \mathrm{~g}, \mathrm{CaCl}_{2} 0,5 \mathrm{~g}$, propilenoglicol $15 \mathrm{~mL}$ em $1.000 \mathrm{~mL}$ de água. Após a administração das soluções eletrolíticas, ocorreu expansão da volemia, em ambos os tratamentos, ocasionando diminuição do hematócrito e proteína total. A SEGli ocasionou o aparecimento de hipernatremia e aumento do lactato plasmático. Por sua vez, a SEProp aumentou a taxa de glicose plasmática, enquanto os demais parâmetros avaliados permaneceram na faixa de referência.
\end{abstract}

Palavras-chave: bovinos, hidratação enteral, soluções eletrolíticas, sonda nasogástrica.

ABSTRACT

This study evaluated the effects of isotonic electrolyte solutions administered via small-bore nasogastric intubation on a continuous flow rate on the hematocrit, total protein, albumin, sodium, potassium, chloride, total magnesium, urea, creatinine, glucose and lactate in cattle experimentally dehydrated. Six crossbred adult cattle, two males and four females were used for the study. Enteral fluid therapy was performed by using $15 \mathrm{~mL} \mathrm{~kg}^{-1} \mathrm{~h}^{-1}$ for 12 hours. The animals were divided in two treatments in a "crossover" experimental design and subjected to the following treatments: SEGlic: $4 \mathrm{~g}$ $\mathrm{NaCl}, 1 \mathrm{~g} \mathrm{KCl}, 4 \mathrm{~g} \mathrm{NaHCO}, 5 \mathrm{~g}$ dextrose in $1.000 \mathrm{~mL}$ of water; SEProp: $8 \mathrm{~g} \mathrm{NaCl}, 0.5 \mathrm{~g} \mathrm{KCl}, 0.5 \mathrm{~g} \mathrm{CaCl}_{2}, 15 \mathrm{~mL}$ propylene glycol in $1.000 \mathrm{~mL}$ of water. Both treatments demonstrated expansion of blood volume, reduction of hematocrit and total protein. In the SEGlic treatment, hypernatremia and increase of lactate were observed. On the other hand, the SEProp treatment caused an increase in the level of plasma glucose, while the other parameters remained within the reference values.

Key words: cattle, enteral fluid therapy, electrolyte solutions, nasogastric tube.

\section{INTRODUÇÃO}

Os desequilíbrios hidro-eletrolíticos e ácido base são frequentemente observados na rotina clínica acompanhando doenças importantes que acometem os bovinos (CONSTABLE, 2003; LISBÔA, 2004). A correção desses desequilíbrios é feita por meio da hidratação, que tem por objetivo a recomposição da volemia e da homeostase (RIBEIRO FILHO et al., 2009).

As vias de administração de soros comumente utilizadas em ruminantes são a intravenosa e a oral. A via intravenosa, por permitir a infusão rápida do volume de reposição, é sempre imprescindível nos casos de desidratação intensa e de choque hipovolêmico. Essa via permite infusão contínua de fluidos, recupera a hidratação, corrige os desequilíbrios eletrolíticos e ácido base, além de aumentar a secreção no trato gastrintestinal. A regra geral para a

'Departamento de Veterinária, Universidade federal de Viçosa (UFV), Avenida P. H. Rolfs, s/n, 36570-000, Viçosa, MG, Brasil. Email: dantas@ufv.br. *Autor para correspondência.

IIDepartamento de Bioquímica e Biologia Molecular, UFV, Viçosa, MG, Brasil. 
administração máxima de fluido por via intravenosa é de 10 a $20 \mathrm{~mL} \mathrm{~kg}^{-1}$ hora $^{-1}$ (SEAHORN \& CORNICKSEAHORN, 1994).

Por questões práticas, no entanto, as infusões intravenosas prolongadas acabam sendo pouco realizadas em ruminantes, em virtude da necessidade de vigilância contínua, contenção apropriada do paciente, complicações com a manutenção da agulha dentro do vaso, hemorragia local e flebite. Além disso, algumas vezes mostra-se de custo elevado, isso porque, para muitas condições clínicas em bovinos, grandes volumes de soro precisam ser administrados (RIBEIRO FILHO et al., 2009). Os fatores de limitação técnica, associados ao valor das soluções comerciais, serviram como estímulo para o incremento do interesse na hidratação por via enteral. De fato, a hidratação enteral, com soluções apropriadas, pode ser considerada, atualmente, a opção de eleição em razão da sua eficácia, praticidade e principalmente pelo custo reduzido, quando comparada à hidratação intravenosa (AVANZA et al., 2004; RIBEIRO FILHO et al., 2007; RIBEIRO FILHO et al., 2009).

A hidratação enteral via sonda nasogástrica de pequeno calibre permite a infusão de soluções eletrolíticas enterais de forma contínua e lenta. Esse procedimento reduz o estresse dos animais, já que minimiza a distensão do rúmen pela administração do soro em bolus e permite a movimentação e alimentação durante a fluidoterapia (RIBEIRO FILHO et al., 2007; AVANZA et al., 2009).

A solução ideal para hidratação é formulada para repor água e eletrólitos de acordo com a necessidade individual do paciente. Muitas vezes, é desejável modificar a composição das soluções baseando-se nos resultados laboratoriais ou nos sinais clínicos, para aumentar o valor terapêutico dessas soluções. Contudo, esse evento nem sempre é possível quando utilizamos a via intravenosa, pois as poucas soluções comerciais de uso intravenoso existentes usualmente não são adequadas para a maioria dos bovinos adultos que necessitam desse recurso terapêutico (ROUSSEL, 1999).

Acredita-se que a composição de soluções eletrolíticas para uso enteral em ruminantes adultos deve conter sódio, cloreto, potássio, cálcio, magnésio e uma fonte de energia, pois, algumas vezes, as enfermidades determinam o aparecimento de inapetência ou anorexia, o que pode gerar o aparecimento de hipoglicemia. Nesses casos, torna-se necessário acrescentar às soluções eletrolíticas substâncias precursoras de energia. Dentre os aditivos gliconeogênicos mais utilizados, destaca-se o propilenoglicol, uma vez que essa substância, quando administrada por via enteral, não sofre ação da microbiota ruminal, sendo absorvida no intestino e transformada em glicose no fígado (STUDER et al., 1993). Contrariamente, a glicose, quando administrada por essa via, é rapidamente metabolizada pela microbiota ruminal (ROUSSEL, 1999).

O presente estudo teve como objetivo avaliar os efeitos de soluções eletrolíticas isotônicas, produzidas artesanalmente e administradas por sonda nasogástrica de pequeno calibre por fluxo contínuo,

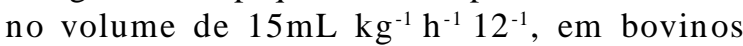
desidratados experimentalmente sobre o hematócrito, proteína total, albumina, sódio, potássio, cloreto, magnésio, uréia, creatinina, glicose e lactato.

\section{MATERIAL E MÉTODOS}

Foram utilizados seis bovinos, dois machos e quatro fêmeas, 18 a 30 meses de idade, sem raça definida, peso corporal médio de $260 \mathrm{~kg}$, clinicamente hígidos. Antes do início do experimento, os animais foram submetidos a exame físico, hemograma, urinálise, perfil bioquímico e pesquisa de hematozoários. Foram alojados em baias individuais arejadas, com cama macia e limpeza diária, alimentados com capim napier picado (Pennisetum purpureum), ração concentrada ${ }^{a}$, fornecida duas vezes ao dia em quantidade equivalente a $1 \%$ do peso corporal e água ad libitum. Junto à ração foram adicionados $50 \mathrm{~g}$ de suplemento mineral ${ }^{\mathrm{b}}$ por dia.

Uma sonda para hidratação enteral, dois metros de comprimento e quatro milímetros de diâmetro, foi introduzida por via nasogástrica e afixada no cabresto dos animais para administração das soluções eletrolíticas. A distribuição inicial dos animais foi aleatória, respeitando-se a ordem até o final do estudo. Os tratamentos foram realizados em dois ciclos, com intervalo de sete dias entre eles. Pelo desenho (Tabela 1), cada tratamento foi constituído por seis animais.

O modelo de desidratação foi desenvolvido para padronização da desidratação com finalidade de avaliar o potencial terapêutico das soluções reidratantes. Foi feito por meio de jejum hídrico e

Tabela 1 - Distribuição dos animais nos tratamentos.

\begin{tabular}{lcc}
\hline Ciclos & Animais & Tratamentos \\
\hline $1^{\text {o }}$ ciclo & $1^{*}, 2^{*}$ e $3^{\mathbf{m}}$ & SEGlic \\
$1^{\circ}$ ciclo & $4^{*}, 5^{*}$, e $6^{\mathbf{m}}$ & SEProp \\
$-2^{\circ}$ ciclo & 4,5 e 6 & SEGlic \\
$2^{\circ}$ ciclo & 1,2, e 3 & SEProp \\
\hline
\end{tabular}

${ }^{*}$ Fêmeas - "Machos

Ciência Rural, v.41, n.2, fev, 2011. 
alimentar durante 24 horas e administração de furosemida ${ }^{c}$, administrada por via intravenosa em duas

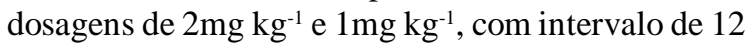
horas. Logo após esse período, iniciaram-se os tratamentos: SEGlic - os animais receberam solução eletrolítica isotônica enteral, constituída por $4 \mathrm{~g} \mathrm{de} \mathrm{NaCl}$, $1 \mathrm{~g}$ de $\mathrm{KCl}, 4 \mathrm{~g}$ de $\mathrm{NaHCO}_{3}$ e $5 \mathrm{~g}$ de dextrose em $1.000 \mathrm{~mL}$ de água (osmolaridade calculada: 287,1 $\mathrm{mosmol} \mathrm{L}^{-1}$ ); SEProp - os animais receberam solução eletrolítica isotônica enteral constituída por $8 \mathrm{~g}$ de $\mathrm{NaCl}, 0,5 \mathrm{~g}$ de $\mathrm{KCl}, 0,5 \mathrm{~g}$ de $\mathrm{CaCl}_{2} 15 \mathrm{~mL}$ de propilenoglicol em $1.000 \mathrm{~mL}$ de água (osmolaridade calculada: $305 \mathrm{mosmol} \mathrm{L}^{-1}$ ). O volume das soluções administradas aos animais de ambos os tratamentos foi $15 \mathrm{~mL} \mathrm{~kg}^{-1} \mathrm{~h}^{-1}$, durante 12 horas, através de sonda nasogástrica ${ }^{\mathrm{d}}$ de pequeno calibre, com $4 \mathrm{~mm}$ de diâmetroe $2 \mathrm{~m}$ de comprimento. A avaliação laboratorial ocorreu nos tempos: T0i imediatamente antes do início da desidratação (0h); T24i - final do período de desidratação (24h); T6t - seis horas de hidratação (6h); T12t - término do período de hidratação (12h); T24f - 12 horas após o término da hidratação (correspondente à fase de avaliação final).

O sangue foi colhido após antissepsia, por venipunção jugular, utilizando-se agulhas e frascos Vacutainer $^{\mathrm{e}}$, contendo EDTA para mensuração do hematócrito. $\mathrm{O}$ hematócrito foi determinado pela técnica do microhematócrito (JAIN, 1986). Alíquotas de soro foram obtidas após centrifugação (3396G) de amostras de sangue colhidas em frascos Vacutainer sem anticoagulante para as determinações de sódio e potássio, por fotometria de chama ${ }^{\mathrm{f}}$ e do cloreto, proteína total, albumina, magnésio total, uréia e creatinina por analisador automático de bioquímica ${ }^{7}$. Para a mensuração da glicose e lactato, as amostras foram colhidas em frascos Vacutainer, contendo fluoreto de sódio para obtenção do plasma e analisadas pelo analisador automático de bioquímicag .

As variáveis quantitativas foram submetidas aos testes de Normalidade (Lilliefors) e Homocedasticidade (Cochran) e posteriormente à análise de variância. Caso apresentasse significância, foi realizado o teste de Tukey (SAEG, 1999).

\section{RESULTADOS E DISCUSSÃO}

$\mathrm{O}$ protocolo de desidratação ocasionou aumento nos valores do hematócrito e das proteínas plasmáticas totais, desidratação discreta a moderada, demonstrando que ele foi eficiente na desidratação dos animais. A utilização de sonda nasogástrica de pequeno calibre em bovinos ainda é pouco descrita na literatura. Nos animais do presente estudo, a sua colocação foi fácil e nenhum deles apresentou intolerância à sonda ou a hidratação. O volume total de soro administrado aos animais variou de 36,9 a 62,1 litros durante 12 horas de hidratação. Todos os animais alimentaram-se, deitaram-se, ruminaram e mantiveram-se alertas durante a hidratação, sugerindo que o estresse causado pela sonda foi mínimo ou desprezível, em concordância com os resultados de AVANZA et al. (2004), ATOJI (2005), RIBEIRO FILHO et al. (2007) e RIBEIRO FILHO et al. (2009).

No presente estudo, o comportamento das curvas das concentrações plasmáticas de proteínas totais e do hematócrito foi semelhante. Ocorreram alterações significativas $(\mathrm{P}<0,05)$ em ambos os grupos, ao longo da fase experimental. Observou-se aumento nos valores do hematócrito e de proteínas totais na fase final de desidratação (T24i). Esse acréscimo foi resultado da desidratação decorrente do jejum hídricoalimentar, associado à administração da furosemida (Tabela 2). Durante a fase de hidratação (T6t e T12t), houve diminuição nos índices do hematócrito e da proteína total, sendo observados os menores valores no T12t (Tabela 1). Esse evento foi ocasionado pela administração da hidratação enteral (HET), a qual determinou a expansão do volume plasmático, como descrito por CONSTABLE et al. (2001), AVANZA et al. (2004) e ATOJI (2005). Não foi detectada diferença entre tratamentos $(\mathrm{P}>0,05)$. Por sua vez, os valores da albumina apresentaram discreto aumento no período de desidratação e diminuição durante a HET em ambos os tratamentos. A causa para os achados observados é a mesma descrita para a proteína total e hematócrito.

Durante a fase de indução da desidratação (T24i), ocorreu aumento do teor do sódio sérico em ambos os tratamentos, decorrente do protocolo de desidratação, entretanto esses valores não foram significativos $(\mathrm{P}>0,05)$. No final da fase de tratamento (T12t), houve apenas aumento nos valores do sódio sérico nos animais do SEglic $(\mathrm{P}<0,05)$, apesar de a composição da solução enteral, utilizada nos animais desse tratamento, ter a metade do cloreto de sódio $(\mathrm{NaCl})$ da que foi utilizada no SeProp. Contudo, há que se considerar que a solução utilizada no SEGlic continha bicarbonato de sódio $\left(\mathrm{NaHCO}_{3}\right)$ e, além disso, esse acréscimo pode ter sido também potencializado pela presença de dextrose, cinco gramas por litro de solução, a qual é benéfica para a absorção de sódio e água, devido ao mecanismo de co-transporte existente no intestino delgado, como citaram AVERY \& SNYDER (1990). Resultados semelhantes foram obtidos por BROOKS et al. (1996), os quais, avaliando soluções eletrolíticas contendo dextrose, administradas por via oral em bezerros, verificaram aumento significativo na concentração de sódio sérico. Porém, CONSTABLE et 
Tabela 2 -Médias e desvios padrão do hematócrito $(\%)$, proteínas totais $\left(\mathrm{g} \mathrm{dL}^{-1}\right)$, albumina $\left(\mathrm{g} \mathrm{dL}^{-1}\right)$, sódio $\left(\mathrm{mEq} \mathrm{L}^{-1}\right)$, potássio $\left(\mathrm{mEq} \mathrm{L}^{-1}\right)$, cloreto $\left(\mathrm{mEq} \mathrm{L}^{-1}\right)$, magnésio $\left(\mathrm{mg} \mathrm{dL}^{-1}\right)$, uréia $\left(\mathrm{mg} \mathrm{dL}^{-1}\right)$, creatinina $\left(\mathrm{mg} \mathrm{dL}^{-1}\right)$, glicose $\left(\mathrm{mg} \mathrm{dL}^{-1}\right)$ e lactato $\left(\mathrm{mmol} \mathrm{L}^{-1}\right)$ de bovinos desidratados experimentalmente e tratados com diferentes soluções eletrolíticas para uso enteral, administradas por sonda nasogástrica de pequeno calibre e fuxo contínuo $\left(15 \mathrm{~mL} \mathrm{~kg}^{-1} 12 \mathrm{~h}^{-1}\right)$.

\begin{tabular}{|c|c|c|c|c|c|}
\hline Tratamento & T0i & $\mathrm{T} 24 \mathrm{i}$ & T6t & $\mathrm{T} 12 \mathrm{t}$ & $\mathrm{T} 24 \mathrm{f}$ \\
\hline & & Hemató & (média \pm desvio-p & - & --o-- \\
\hline SEGlic & $25,80 \pm 4,27^{\mathrm{Ab}}$ & $30,40 \pm 4,56^{\mathrm{Aa}}$ & $26,40 \pm 4,04^{\mathrm{Ab}}$ & $25,60 \pm 3,65^{\mathrm{Ab}}$ & $25,80 \pm 3,77^{\mathrm{Ab}}$ \\
\hline \multirow[t]{2}{*}{ SEProp } & $25,20 \pm 5,81^{\text {Aab }}$ & $28,20 \pm 5,22^{\mathrm{Aa}}$ & $26,20 \pm 5,12^{\mathrm{Aab}}$ & $24,80 \pm 5,54^{\mathrm{Ab}}$ & $26,00 \pm 4,69^{\mathrm{Aab}}$ \\
\hline & & \multicolumn{4}{|c|}{-- Proteínas totais g dL ${ }^{-1}$ (média \pm desvio-padrão) } \\
\hline SEGlic & $7,80 \pm 0,64^{\mathrm{Ab}}$ & $8,62 \pm 0,52^{\mathrm{Aa}}$ & $8,01 \pm 0,49^{\mathrm{Aab}}$ & $7,62 \pm 0,58^{\mathrm{Ab}}$ & $8,07 \pm 0,3^{\text {Aab }}$ \\
\hline \multirow[t]{2}{*}{ SEProp } & $7,96 \pm 0,41^{\mathrm{Ab}}$ & $9,0 \pm 0,93^{\mathrm{Aa}}$ & $8,54 \pm 0,55^{\mathrm{Aab}}$ & $8,02 \pm 0,36^{\mathrm{Ab}}$ & $8,35 \pm 0,61^{\mathrm{Aab}}$ \\
\hline & & \multicolumn{4}{|c|}{ Albumina g L ${ }^{-1}$ (média \pm desvio-padrão) } \\
\hline SEGlic & $3,61 \pm 0,28^{\mathrm{Aa}}$ & $3,79 \pm 0,21^{\text {Aa }}$ & $3,63 \pm 0,20^{\mathrm{Aa}}$ & $3,59 \pm 0,24^{\mathrm{Aa}}$ & $3,59 \pm 0,32^{\mathrm{Aa}}$ \\
\hline \multirow[t]{2}{*}{ SEProp } & $3,64 \pm 0,25^{\mathrm{Ab}}$ & $3,93 \pm 0,32^{\mathrm{Aa}}$ & $3,69 \pm 0,24^{\mathrm{Aab}}$ & $3,62 \pm 0,23^{\mathrm{Ab}}$ & $3,70 \pm 0,24^{\mathrm{Aab}}$ \\
\hline & & \multicolumn{4}{|c|}{ 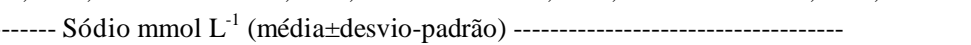 } \\
\hline SEGlic & $145,22 \pm 4,46^{\mathrm{Ab}}$ & $150,70 \pm 7,13^{\mathrm{Ab}}$ & $160,78 \pm 4,54^{\text {Aab }}$ & $163,65 \pm 7,93^{\mathrm{Aa}}$ & $152,25 \pm 6,02^{\mathrm{Ab}}$ \\
\hline \multirow[t]{2}{*}{ SEProp } & $143,90 \pm 10,12^{\mathrm{Aa}}$ & $148,70 \pm 2,13^{\mathrm{Aa}}$ & $139,82 \pm 23,25^{\text {Aa }}$ & $139,13 \pm 20,58^{\text {Aa }}$ & $140,35 \pm 15,15^{\mathrm{Aa}}$ \\
\hline & & \multicolumn{4}{|c|}{--- Potássio mmol L'1 (média \pm desvio-padrão) -- } \\
\hline SEGlic & $5,44 \pm 0,28^{\mathrm{Aa}}$ & $5,27 \pm 0,84^{\mathrm{Aa}}$ & $5,19 \pm 0,53^{\mathrm{Aa}}$ & $5,25 \pm 0,68^{\mathrm{Aa}}$ & $5,21 \pm 0,49^{\mathrm{Aa}}$ \\
\hline \multirow[t]{2}{*}{ SEProp } & $5,60 \pm 0,16^{\mathrm{Aa}}$ & $5,31 \pm 0,79^{\mathrm{Aa}}$ & $5,17 \pm 0,79^{\mathrm{Aa}}$ & $5,07 \pm 0,21^{\mathrm{Aa}}$ & $5,54 \pm 0,36^{\mathrm{Aa}}$ \\
\hline & & \multicolumn{4}{|c|}{ 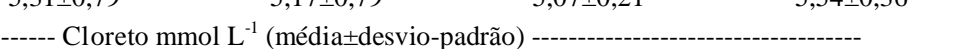 } \\
\hline SEGlic & $97,54 \pm 1,47^{\mathrm{Aa}}$ & $93,96 \pm 1,09^{\mathrm{Aa}}$ & $96,42 \pm 1,09^{\mathrm{Aa}}$ & $96,92 \pm 2,80^{\mathrm{Aa}}$ & $96,48 \pm 1,69^{\mathrm{Aa}}$ \\
\hline \multirow[t]{2}{*}{ SEProp } & $98,04 \pm 2,59^{\mathrm{Aa}}$ & $94,52 \pm 0,15^{\mathrm{Aa}}$ & $98,80 \pm 2,16^{\mathrm{Aa}}$ & $102,56 \pm 2,73^{\mathrm{Ba}}$ & $101,86 \pm 1,60^{\mathrm{Ba}}$ \\
\hline & & \multicolumn{4}{|c|}{ 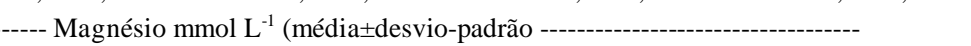 } \\
\hline SEGlic & $1,90 \pm 0,26 \mathrm{Aa}$ & $2,04 \pm 0,12 \mathrm{Aa}$ & $1,95 \pm 0,15 \mathrm{Aa}$ & $1,86 \pm 0,31 \mathrm{Aa}$ & $1,87 \pm 0,32 \mathrm{Aa}$ \\
\hline \multirow[t]{2}{*}{ SEProp } & $1,84 \pm 0,25 \mathrm{Aa}$ & $1,98 \pm 0,28 \mathrm{Aa}$ & $1,85 \pm 0,17 \mathrm{Aa}$ & $1,72 \pm 0,21 \mathrm{Aa}$ & $1,81 \pm 0,32 \mathrm{Aa}$ \\
\hline & & \multicolumn{4}{|c|}{ - Uréia (média士desvio-padrão) --- } \\
\hline SEGlic & $3,33 \pm 4,69^{\mathrm{Ab}}$ & $20,64 \pm 6,22^{\mathrm{Aa}}$ & $23,56 \pm 5,90^{\mathrm{Aa}}$ & $4,21 \pm 4,93^{\mathrm{Ab}}$ & $3,76 \pm 4,30^{\mathrm{Ab}}$ \\
\hline \multirow[t]{2}{*}{ SEProp } & $3,94 \pm 2,10^{\mathrm{Ab}}$ & $13,51 \pm 2,98^{\mathrm{Aa}}$ & $18,86 \pm 5,01^{\mathrm{Aa}}$ & $5,43 \pm 5,24^{\mathrm{Ab}}$ & $3,22 \pm 0,48^{\mathrm{Ab}}$ \\
\hline & & \multicolumn{4}{|c|}{ Creatinina (média \pm desvio-padrão) } \\
\hline SEGlic & $0,54 \pm 0,2^{\mathrm{Aa}}$ & $0,74 \pm 0,22^{\mathrm{Aa}}$ & $0,66 \pm 0,19^{\mathrm{Aa}}$ & $0,69 \pm 0,24^{\mathrm{Aa}}$ & $0,59 \pm 0,22^{\mathrm{Aa}}$ \\
\hline \multirow[t]{2}{*}{ SEProp } & $0,67 \pm 0,24^{\mathrm{Aa}}$ & $0,78 \pm 0,28^{\mathrm{Aa}}$ & $0,80 \pm 0,23^{\mathrm{Aa}}$ & $0,87 \pm 0,26^{\mathrm{Aa}}$ & $0,63 \pm 0,11^{\mathrm{Aa}}$ \\
\hline & & \multicolumn{4}{|c|}{ 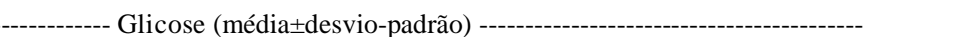 } \\
\hline SEGlic & $90,52 \pm 2,90^{\mathrm{Aa}}$ & $88,02 \pm 6,83^{\mathrm{Aa}}$ & $79,60 \pm 10,23^{\text {Аa }}$ & $90,60 \pm 5,33^{\mathrm{Aa}}$ & $85,74 \pm 3,59^{\mathrm{Aa}}$ \\
\hline \multirow[t]{2}{*}{ SEProp } & $78,26 \pm 5,83^{\mathrm{Ab}}$ & $76,54 \pm 8,74^{\mathrm{Ab}}$ & $87,82 \pm 7,30^{\text {Aab }}$ & $97,08 \pm 13,65^{\mathrm{Aa}}$ & $91,84 \pm 13,76^{\mathrm{Aa}}$ \\
\hline & & \multicolumn{4}{|c|}{---- Lactato (média } \\
\hline SEGlic & $1,18 \pm 0,72^{\mathrm{Aa}}$ & $2,38 \pm 1,32^{\mathrm{Aa}}$ & $1,28 \pm 0,65^{\mathrm{Aa}}$ & $2,39 \pm 1,24^{\mathrm{Aa}}$ & $1,62 \pm 0,75^{\mathrm{Aa}}$ \\
\hline SEProp & $1,22 \pm 0,39^{\mathrm{Aa}}$ & $2,27 \pm 1,97^{\mathrm{Aa}}$ & $1,51 \pm 0,82^{\mathrm{Aa}}$ & $1,39 \pm 0,62^{\mathrm{Aa}}$ & $0,77 \pm 0,10^{\mathrm{Aa}}$ \\
\hline
\end{tabular}

Análise de variância (medidas repetidas).

As médias na mesma coluna, seguidas por letras maiúsculas diferentes, e as médias na mesma linha, seguidas por letras minúsculas diferentes, diferem pelo teste de Tukey $(\mathrm{P}<0,05)$.

T0i (imediatamente antes do início da hidratação), T24i (24 horas antes do início da hidratação), T6t (seis horas de hidratação), T12t (12 horas de hidratação) e T24f (doze horas após o término da hidratação).

Tratamento SEGlic - a solução foi preparada utilizando-se $4 \mathrm{~g}$ de $\mathrm{NaCl}, 1 \mathrm{~g}$ de $\mathrm{KCl}, 4 \mathrm{~g}$ de $\mathrm{NaHCO}_{3}$ e $5 \mathrm{~g}$ de dextrose em $1.000 \mathrm{~mL}$ de água (osmolaridade calculada: 283,1 mosmol L $\mathrm{L}^{-1}$ ); Tratamento SEProp - a solução foi preparada adicionando $8 \mathrm{~g} \mathrm{de} \mathrm{NaCl}, 0,5 \mathrm{~g} \mathrm{de} \mathrm{KCl}, 0,5 \mathrm{~g}$ de $\mathrm{CaCl}_{2}$ e $15 \mathrm{~mL}$ de propilenoglicol em $1.000 \mathrm{~mL}$ da solução eletrolítica (osmolaridade calculada: $305 \mathrm{mosmol} \mathrm{L}^{-1}$ ).

al. (2001), comparando duas soluções enterais contendo glicose em bezerros desidratados, não observaram aumento na concentração de sódio sérico durante a hidratação.

Os valores de potássio não foram alterados durantes toda a fase experimental em ambos os tratamentos $(\mathrm{P}>0,05)$, estando em concordância com os obtidos por ATOJI (2005). O teor do cloreto sérico na fase de indução da desidratação (T24i) apresentou diminuição nos dois tratamentos, ocasionado pela administração da furosemida, como descrito por FREESTONE et al. (1988) e ALVES et al. (2005), entretanto, esse decréscimo não foi significativo $(P>0,05)$. Diminuições nos valores do cloreto não foram 
observadas por CONSTABLE et al. (2001), ATOJI (2005) e AVANZA et al. (2009). No presente estudo, a administração da hidratação enteral não ocasionou aumento nos valores do cloreto sérico ao longo da fase experimental nos dois tratamentos. Como expresso na tabela 2, detectou-se diferença entre tratamentos no T12t e T24f. Os valores dos animais do tratamento SEProp foram superiores $(\mathrm{P}<0,05)$ aos do tratamento SEGlic. Essa diferença foi determinada pela composição da solução contendo propilenoglicol, a qual continha mais cloreto. Entretanto, convém ressaltar que os valores do cloreto no tratamento SEProp encontravamse na faixa de referência que, segundo KANEKO et al. (1997), é de 97 a $111 \mathrm{mmol} \mathrm{L}^{-1}$, o que torna esse resultado sem significado clínico.

No presente ensaio, observou-se aumento $(\mathrm{P}<0,05)$ nos valores de uréia durante a fase de desidratação (T24i), em ambos os tratamentos, decorrente da hipovolemia. Os valores continuaram elevados durantes as primeiras seis horas de hidratação (T6t), com diminuição $(\mathrm{P}<0,05)$ no final da fase de reidratação (T12t). O aumento da concentração da uréia durante a fase de desidratação era esperada, devido ao menor fluxo sanguíneo renal e diminuição na taxa de filtração glomerular, levando a azotemia pré-renal. Resultados semelhantes foram encontrados por CONSTABLE et al. (2001) e ATOJI (2005). Já a diminuição, a partir do T12t (Tabela 2), foi devido ao efeito da hidratação enteral, levando à expansão do volume plasmático dos animais desidratados, corroborando os resultados descritos por CONSTABLE et al. (2001), ATOJI (2005), ALVES et al. (2005) e AVANZA et al. (2009).

Os valores da creatinina e do magnésio total não apresentaram alterações significativas ao longo do tempo e entre os tratamentos $(\mathrm{P}>0,05)$, como demonstrado na tabela 2. Pode-se constatar, na referida tabela, que os valores da glicose plasmática não tiveram alterações durante toda a fase experimental nos animais do tratamento SEGlic $(\mathrm{P}>0,05)$. Por outro lado, nos animais que receberam SEProp, solução contendo propilenoglicol, houve aumento $(\mathrm{P}<0,05)$ na concentração da glicose a partir da sexta hora de tratamento (T6t), atingindo os maiores valores no final da hidratação (T12t), mantendo-se elevados até o momento da avaliação final (T24f). O constituinte da solução enteral no tratamento SEProp responsável pelo aumento da glicose plasmática foi o propilenoglicol, que, administrado via enteral em ruminantes, escapa intacto da fermentação ruminal e, quase na sua totalidade, é absorvido no intestino e transformado em glicose no fígado como citou STUDER et al. (1993). Avaliando o efeito de drench contendo propilenoglicol em vacas no pré-parto e no pós-parto, STUDER et al. (1993) e MIYOSHI et al. (2001), respectivamente, constataram aumento na concentração da glicose plasmática. No presente ensaio, o componente energético utilizado no tratamento SEGlic, glicose, na dose de $5 \mathrm{~g}$ por litro de solução, não apresentou efeito sobre a glicemia, sinalizando que a utilização dessa substância em soluções eletrolíticas administradas por via enteral em bovinos adultos não apresenta o efeito desejado.

Os valores do lactato plasmático não apresentaram alterações $(\mathrm{P}>0,05)$ durante toda a fase experimental em ambos os tratamentos (Tabela 2). Mesmo não apresentando valores significativos, observa-se que, nos animais do tratamento SEGlic, as concentrações do lactato foram superiores $(92,6 \%)$ aos dos animais do tratamento SEProp no final da hidratação enteral (T12t). Esse aumento é atribuído à presença de glicose na solução hidratante, que é fermentada pela microbiota ruminal a D-lactato, sendo prontamente absorvida pela parede ruminal, aumentando os níveis plasmáticos desse elemento.

\section{CONCLUSÃO}

A velocidade de infusão de $15 \mathrm{~mL} \mathrm{~kg}^{-1} \mathrm{~h}^{-1}$ durante 12 horas determina a expansão da volemia, expressa pela diminuição do hematócrito e da proteína total. A SEGli ocasiona o aparecimento de hipernatremia e aumento do lactato plasmático. Por sua vez, a SEProp aumenta a taxa de glicose plasmática, enquanto os demais parâmetros permanecem na faixa de referência, demonstrando que a SEProp é mais adequada para o uso em bovinos adultos quando se deseja fornecer energia através de soluções eletrolíticas por via enteral.

\section{FONTES DE AQUISIÇÃO}

a - Ração para bovinos - Fábrica de ração da Universidade Federal de Viçosa (UFV).

b - Suplemento Mineral Purina.

c - Urolab - Marcolab.

d - Sondas fabricadas no Setor de Clínica e Cirurgia de Grandes Animais - DVT/UFV.

e - Vacutainer BD - Becton \& Dickinson Ind. Cirúrgica Ltda., Brasil.

f - Fotômetro de chama B462 - Micronal.

g - Analizador Automático de Bioquímica - Alizè - França.

Foi submetido à Comissão de Ética e Biossegurança do DVT/ UFV. Protocolo 31/2005.

\section{REFERÊNCIAS}

ALVES, G.E.S. et al. Tratamento da compactação experimental do cólon maior em equinos: resultados de laboratório e exames bioquímicos. Arquivo Brasileiro de Medicina Veterinária 
e Zootecnia, v.57, p.238-287, 2005. Disponível em: <http:/ / www.scielo.br/scielo.php?pid=S0102...script>. Acesso em 02 mar. 2006. doi: 10.1590/S0102-09352005000300001.

ATOJI, K. Fluidoterapia por via nasogástrica em caprinos. 2005. 109f. Dissertação (Mestrado em Medicina Veterinária) - Curso de Pós-graduação em Medicina Veterinária, Universidade Federal de Viçosa, MG.

AVANZA, M.F.B. et al. Fluidoterapia enteral em vacas normais e experimentalmente desidratadas. In: COMBRAVET, 2004, São Luís, MA. Anais... São Luís: Universitária, 2004. p.31.

AVANZA, M.F.B. et al. Hidratação enteral em equinos - solução eletrolítica associada ou não à glicose, à maltodextrina e ao sulfato de magnésio: resultado de laboratório. Ciência Rural, v.39, p.1126-1133, 2009. Disponível em: <http:// www.scielo.br/scielo.php?script=sci_arttext\&pid $>$. Acesso em 10 mar. 2010. doi: 10.1590/S0103-84782009005000021.

AVERY, M.E.; SNYDER, J.D. Oral therapy for acute diarrhea. New England Journal of Medicine, v.323, p.891-894, 1990.

BROOKS, H.W. et al. Fallibility of faecal consistency as a criterion of success in the evaluation of oral fluid therapy for calf diarrhea. British Veterinary Journal, v.153, p.75-81, 1996.

CONSTABLE, P.D. Fluid and electrolyte therapy in ruminants. Veterinary Clinical of North America Food Animal Practice, v.19, p.557-597, 2003.

CONSTABLE, P.D. et al. Comparison of two oral electrolyte solutions for the treatment of dehydrated calves with experimentally-induced diarrhea. Veterinary Journal, v.162, p.129-140, 2001.

FREESTONE J.F. et al. Influence of furosemide treatment on fluid and electrolyte balance in the horse. American biochemistry of Veterinary Research, v.49, p.1899-1902, 1988 .
JAIN, N.C. Veterinary hematology. 4.ed. Philadelphia: Lea \& Febier, 1986. 1221p.

KANEKO J.R. et al. Clinical biochemistry of domestic animal. 5.ed. San Diego: Academic, 1997. 932p.

LISBÔA, J.A.N. Fluidoterapia em ruminantes: uma abordagem prática. In: CONGRESSO PAULISTA DE MEDICINA VETERINÁRIA, 2004, Santos, SP. Anais... Santos: CRMVSP, 2004. p.3-6

MIYOSHI, S. Effects of propylene glycol drenching on energy balance, plasma glucose, plasma insulin, ovarian function and conception in dairy cows. Animal Reproduction Science, v.68, p.29-43, 2001.

RIBEIRO FILHO, J.D. et al. Hidratação enteral em bovinos via sonda nasogástrica por fluxo contínuo. Ciência Animal Brasileira, v.11, Supl, p.24-28, 2009.

RIBEIRO FILHO, J.D. et al. Tratamento de bovinos desidratados experimentalmente com soluções eletrolíticas por via enteral administradas por sonda nasogástrica. Archives of Veterinary Science, v.12, Supl, p.49, 2007.

ROUSSEL, A.J. Fluid therapy in mature cattle. Veterinary Clinics of North America Food Animal Practice, v.15, p.545-557, 1999.

SEAHORN, T.T.; CORNICK-SEAHORN, J. Fluid therapy. Veterinay Clinics of North America Equine Practice, v.10, p.517-525, 1994 .

SISTEMA DE ANÁLISE ESTATÍSTICA E GENÉTICA (SAEG), UFV. Central de processamento de dados. Viçosa, 1999. $285 \mathrm{p}$.

STUDER, V.A. et al. Effect of prepartum propylene glycol administration on periparturient fatty liver in dairy cows. Journal Dairy Science, v.76, p.2931-2939, 1993. 